\title{
THE SHARP WEIGHTED BOUND FOR THE RIESZ TRANSFORMS
}

\author{
STEFANIE PETERMICHL
}

(Communicated by Michael Lacey)

\begin{abstract}
We establish the best possible bound on the norm of the Riesz transforms as operators in the weighted space $L_{\mathbb{R}^{n}}^{p}(\omega)$ for $1<p<\infty$ in terms of the classical $A_{p}$ characteristic of the weight.
\end{abstract}

\section{INTRODUCTION}

A weight $\omega$ is a positive $L_{l o c}^{1}$ function. Muckenhoupt proved in [9] that the maximal function is bounded on $L^{p}(\omega)$ for $1<p<\infty$ if and only if the weight $\omega$ belongs to the class $A_{p}$. Here $\omega \in A_{p}$ if and only if

$$
Q_{p}(\omega):=\sup _{I}\langle\omega\rangle_{I}\left\langle\omega^{-1 /(p-1)}\right\rangle_{I}^{p-1}<\infty .
$$

The notation $\langle\cdot\rangle_{I}$ denotes the average over the interval $I$ and the supremum runs over all intervals $I$. Hunt, Muckenhoupt, and Wheeden proved in [7] that the $A_{p}$ condition also characterizes the boundedness of the Hilbert transform in $L^{p}(\omega)$. Coifman and Fefferman in [3] extended the theory to general Calderón-Zygmund operators.

It is of special interest to find sharp bounds for the operator norms in terms of the $A_{p}$ characteristic $Q_{p}(\omega)$ of the weight. The aim is on controlling the operator norm by a suitable function of the $A_{p}$ characteristic of $\omega$. One seeks to prove an estimate of the form

$$
\|T f\|_{L^{p}(\omega)} \lesssim N_{p}\left(Q_{p}(\omega)\right)\|f\|_{L^{p}(\omega)}
$$

for a fixed operator $T$ with a suitable increasing function $N_{p}$, where the implied constant is independent of $f$ or $\omega$. Since $Q_{p}(\omega) \geq 1$, it is desirable to find for example estimates with $N_{p}(x)=x^{r}$ with $r=r(p)$ as small as possible.

Such estimates have applications in PDE; see for example in the case of the Hilbert transform, the work by Fefferman, Kenig, and Pipher in [5]. More recently, Volberg and the author solved a known problem related to quasiregular maps through a sharp bound for the weighted Beurling operator. See [1] and [14]. To solve the borderline case of a regularity problem, the exact sharp growth of the Beurling-Ahlfors transform in $L^{p}(\omega)$ was needed while the other cases could be handled merely using any bound for the operator in the weighted space.

Received by the editors September 19, 2006.

2000 Mathematics Subject Classification. Primary 42-XX.

The author was supported by NSF grant \#DMS 9729992.

(C) 2007 American Mathematical Society Reverts to public domain 28 years from publication 
The present work is concerned with the sharp bound for the operator norm of the Riesz transforms in $L^{2}(\omega)$. We then derive the sharp bound for other $p$ by means of extrapolation. S. Buckley proved in [2] an $L^{2}$ bound in terms of the square of the classical $A_{2}$ characteristic of the weight. Recently, A. Lerner established in [8] an improvement to $3 / 2$. In this text, we establish the linear and best possible bound.

As for other Calderón-Zygmund operators, the sharp bound is only known for the Beurling operator (see [14]) the squares of Riesz transforms, implicit in [14], and the Hilbert transform (see [12]). To obtain the sharp estimates is of course significantly more difficult than to just prove continuity. In the weighted situation, it essentially amounts to solving a two-weight question as a separation in the relationship of the weights ensures that nothing is lost in the estimate of the norm. Naively, if one uses the fact that $w v=1$ as for $v=w^{-1}$ is the case, one loses enough to end up with a less than optimal bound. Currently, all proofs that yield optimal bounds rely in their core on martingale inequalities or estimates for other dyadic operators that also act locally on the Haar system - this text is no exception. The way in which the dyadic estimates are used though varies greatly.

It is well understood that Haar multipliers and martingales serve as a good model for singular integral operators, but they appear to be a much better model for even operators, such as the Beurling operator rather than for odd ones such as the Hilbert and Riesz transforms. In [11] the author introduced new dyadic model operators that seem better suited for antisymmetric operators. The Hilbert transform can essentially be expressed as an average of these operators, called dyadic shifts. This technique then was extended to the Riesz transforms in [13], using the product Haar system over cubes in $\mathbb{R}^{n}$. We find it necessary to switch to a modified product Haar system because of some orthogonality issues that appear in the weighted situation when the dimension is greater than 1 .

This approach reduces these continuous problems to dyadic ones, where Bellman functions, as introduced to harmonic analysis in [10], have proven to be highly effective. However, this technique is very delicate and not very well suited to estimate the antisymmetric shift operators. To do so, one needs to invent a trick to deal with a certain difficulty induced by the shift that does not appear while estimating Haar multipliers. This was accomplished in one dimension in [12]. In the present text we give an estimate for the $n$-dimensional analog of the shift operator and hence establish the sharp weighted bound for the family of Riesz transforms. The proof involves a passage in the technique of Bellman functions to several variables. One loses the martingale structure, which by itself causes little difficulty. However, one needs to overcome an $n$-dimensional version of a difficulty induced by the shift operator. To do so, it is necessary to adjust the Bellman function and increase the number of variables with the dimension.

\section{MAIN RESULTS AND DEFINITIONS}

We consider spaces $L_{\mathbb{R}^{n}}^{p}(\omega)$ for $1<p<\infty$, where $\omega$ is a positive $L_{\text {loc }}^{1}$ function, called a weight. Let $d A(x)$ be Lebesgue measure on $\mathbb{R}^{n}$. The norm of $f \in L_{\mathbb{R}^{n}}^{p}(\omega)$ is

$$
\|f\|_{L^{p}(\omega)}:=\left(\int_{\mathbb{R}^{n}}|f(x)|^{p} \omega(x) d A(x)\right)^{1 / p} .
$$


We are concerned with a special class of weights, called $A_{p}$. We say $\omega \in A_{p}$ if

$$
Q_{p}(\omega):=\sup _{Q}\langle\omega\rangle_{Q}\left\langle\omega^{-\frac{1}{p-1}}\right\rangle_{Q}^{p-1}<\infty,
$$

where the supremum runs over cubes in $\mathbb{R}^{n}$ and the notation $\langle\cdot\rangle_{Q}$ is used for the average over $Q$ with respect to Lebesgue measure $\langle f\rangle_{Q}:=\frac{1}{|Q|} \int_{Q} f$. The symbol $R_{k}$ stands for the $k^{\text {th }}$ direction Riesz transform on $\mathbb{R}^{n}$ and is defined by its Fourier multiplier as follows:

$$
\hat{R_{k}} f(\xi)=i \frac{\xi_{k}}{|\xi|} \hat{f}(\xi)
$$

Here is our main theorem:

Theorem 2.1. There exists a constant c so that for all weights $\omega \in A_{2}$ the Riesz transforms as operators in weighted space $R_{k}: L_{\mathbb{R}^{n}}^{2}(\omega) \rightarrow L_{\mathbb{R}^{n}}^{2}(\omega)$ have operator norm $\left\|R_{k}\right\| \leq c Q_{2}(\omega)$ and this result is sharp.

The proof relies both on the decomposition of the Riesz transforms into dyadic operators and a modification of the Bellman function technique.

We also prove the following more general version:

Corollary 2.2. For $1<p<\infty$ there exists $c$ only depending on $p$ and $n$ so that $R_{k}$ : $L_{\mathbb{R}^{n}}^{p}(\omega) \rightarrow L_{\mathbb{R}^{n}}^{p}(\omega)$ have operator norm $\left\|R_{k}\right\| \leq c Q_{p}(\omega)^{r}$, where $r=\max \left\{1, p^{\prime} / p\right\}$ and this is sharp.

\section{NotATION}

We use a convenient notation for unweighted averages with respect to Lebesgue measure and also for weighted averages

$$
\langle f\rangle_{Q}=\frac{1}{|Q|} \int_{Q} f \text { and }\langle f\rangle_{Q, \omega}:=\frac{1}{\omega(Q)} \int_{Q} f \omega
$$

where $\omega(Q)=\int_{Q} \omega$. By $(f, g)$ we mean the standard real inner product in $L^{2}$, by $(f, g)_{\omega}$ we mean the weighted inner product $(f, g)_{\omega}:=\int_{\mathbb{R}^{n}} f g \omega$. Let $\mathcal{D}$ denote the collection of all dyadic intervals in $\mathbb{R}$, that is, the collection of intervals $\mathcal{D}=$ $\left\{\left[n 2^{k},(n+1) 2^{k}\right]: n, k \in \mathbb{Z}\right\}$. We call $\mathcal{D}$ the standard dyadic grid in $\mathbb{R}$. We consider the grid $\mathcal{D}^{n}$ in $\mathbb{R}^{n}$, consisting of cubes $Q=I_{1} \times \ldots \times I_{n}$ with $I_{i}$ all of the same length. For each $\alpha \in \mathbb{R}, r>0$, let us consider the translated dilate of the standard dyadic grid in one variable, which is $\mathcal{D}^{\alpha, r}=\{\alpha+r I: I \in \mathcal{D}\}$. In $\mathbb{R}^{n}$ we will also find it necessary to consider rotations of the grid. We build the standard grid in $\mathbb{R}^{n}$, rotate all cubes by rotation $\rho$, then dilate them by $r>0$ and finally consider translations along $\vec{\alpha} \in \mathbb{R}^{n}$.

Each one-dimensional dyadic grid gives rise to an orthonormal system in $L^{2}$, the Haar system $\left\{h_{I}^{0}: I \in \mathcal{D}\right\}$ where $h_{I}^{0}=|I|^{-1 / 2}\left(\chi_{I_{+}}-\chi_{I_{-}}\right)$. By $I_{+}$we mean the right half of $I$, and by $I_{-}$we mean the left half. It will also be convenient to consider $L^{2}$-normalized indicator functions $h_{I}^{1}=|I|^{-1 / 2} \chi_{I}$. In $\mathbb{R}^{n}$, there are many different choices for orthonormal bases based on the Haar system. We find it necessary to use a variation of the standard product Haar system, due to an issue that arises with weights when $n \geq 2$. The following system will be the most convenient for us. Let us enumerate the directions by $1, \ldots, n$ and let us define the set of signatures as $\left\{\sigma_{0}\right\} \cup \bigcup_{k=1}^{n-1}\{+,-\}^{\{1, \ldots, k\}}$. To $\sigma_{0}$ we associate the Haar function $h_{I_{1}}^{0} \times h_{I_{2}}^{1} \times \ldots \times h_{I_{n}}^{1}$ and for all other signatures we proceed as follows. If the signature is of length $s$, say 
$\sigma=\left(\sigma_{1}, \ldots, \sigma_{s}\right)$, a sequence of \pm , then take $h_{I_{1} \sigma_{1}}^{1} \times \ldots \times h_{I_{s} \sigma_{s}}^{1} \times h_{I_{s+1}}^{0} \times h_{I_{s+2}}^{1} \times \ldots \times h_{I_{n}}^{1}$, where $h_{I_{k}+}^{1}$ is the normalized indicator on the right hand side of $I_{k}$. This splits the dyadic cube $I_{1} \times \ldots \times I_{n}$ into pieces that are associated with,+- or 0 . For $n=2$ and $Q=I_{1} \times I_{2}$ these are the functions:
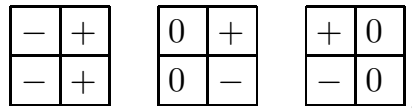

Let us introduce an appropriate orthonormal system for $L^{2}(\omega)$, the unbalanced Haar system. Note that each Haar function is made up of boxes that are associated with a,+- , or 0 . Let $Q_{+(\sigma)}$ be the box that is associated with + and $Q_{-(\sigma)}$ analogously. These of course will depend upon the signature $\sigma$, as indicated. Define

$$
h_{\omega, Q}^{\sigma}:=\frac{1}{\omega\left(Q_{+(\sigma)} \cup Q_{-(\sigma)}\right)^{1 / 2}}\left[\frac{\omega\left(Q_{-(\sigma)}\right)^{1 / 2}}{\omega\left(Q_{+(\sigma)}\right)^{1 / 2}} \chi_{Q_{+(\sigma)}}-\frac{\omega\left(Q_{+(\sigma)}\right)^{1 / 2}}{\omega\left(Q_{-(\sigma)}\right)^{1 / 2}} \chi_{Q_{-(\sigma)}}\right] .
$$

Note that $h_{\omega, Q}^{\sigma}$ is orthogonal to functions that are constant on $Q_{-(\sigma)} \cup Q_{+(\sigma)}$ in $L^{2}(\omega)$ and that it is complete. For $Q \supsetneq Q^{\prime}, h_{Q}^{\omega}$ is constant on $Q^{\prime}$. We denote this constant by $h_{\omega, Q}^{\sigma}\left(Q^{\prime}\right)$. One can see that we obtain the weighted average of $g$ over $Q^{\prime}$ when only summing over superintervals of $Q^{\prime}$ :

$$
\sum_{Q: Q \supsetneq Q^{\prime}} \sum_{\sigma}\left(g, h_{\omega, Q}^{\sigma}\right)_{\omega} h_{\omega, Q}^{\sigma}\left(Q^{\prime}\right)=\langle g\rangle_{Q^{\prime}, \omega} .
$$

\section{Reduction to DyAdic sums}

Let $Q \in \mathcal{D}^{\vec{\alpha}, r, \rho}$ and let $Q_{0}$ be a child of $Q$. Also fix the signature $\sigma_{0}$. By [13], we have the following theorem, which is key to our proof:

Theorem 4.1. A non-zero multiple of $R_{i}$ lies in the closed convex hull of a linear combination of operators densely defined by

$$
\amalg^{\vec{\alpha}, r, \rho} h_{Q}^{\sigma_{0}}=h_{Q_{0}}^{\sigma_{0}} \text { (reads "sha") and } \amalg^{\vec{\alpha}, r, \rho} h_{Q}^{\sigma}=0 \text { for } \sigma \neq \sigma_{0} .
$$

These operators are referred to as dyadic shifts. In [13], the standard product Haar system was used; however, there is no difference if we define the shift operator as above. The different Haar systems can be easily expressed by one another. It is therefore only necessary to prove the desired bound uniformly for all dyadic shifts as described above and hence the bound for the Riesz transforms will follow. We omit the indices $\rho, \vec{\alpha}$ and $r$ because all our estimates are uniform. We have to obtain the estimate $\|\amalg\|_{L^{2}(\omega) \rightarrow L^{2}(\omega)} \lesssim Q_{2}(\omega)$. By duality, this is equivalent to $\left|\left(\amalg_{\omega^{-1}} f, g\right)_{\omega}\right| \lesssim Q_{2}(\omega)\|f\|_{\omega^{-1}}\|g\|_{\omega}$ for positive test functions $f$ and $g$. Positivity of $f$ and $g$ will make the domain for the Bellman function slightly more convenient. The operator $\amalg_{\omega^{-1}}$ is here the composition of $\amalg$ with multiplication by $\omega^{-1}$, that is $\amalg_{\omega^{-1}}(f)=\amalg\left(\omega^{-1} f\right)$. Expanding $f$ and $g$ in the unbalanced Haar systems for $L^{2}\left(\omega^{-1}\right)$ and $L^{2}(\omega)$, respectively, yields

$$
\left(\amalg_{\omega^{-1}} f, g\right)_{\omega}=\sum_{Q, Q^{\prime}} \sum_{\sigma, \sigma^{\prime}}\left(f, h_{\omega^{-1}, Q^{\prime}}^{\sigma^{\prime}}\right)_{\omega^{-1}}\left(g, h_{\omega, Q}^{\sigma}\right)_{\omega}\left(\amalg_{\omega^{-1}} h_{\omega^{-1}, Q^{\prime}}^{\sigma^{\prime}}, h_{\omega, Q}^{\sigma}\right)_{\omega} .
$$

Here

$$
\left(\amalg_{\omega^{-1}} h_{\omega^{-1}, Q^{\prime}}^{\sigma^{\prime}}, h_{\omega, Q}^{\sigma}\right)_{\omega}=\sum_{L}\left(h_{L}^{\sigma_{0}}, h_{\omega^{-1}, Q^{\prime}}^{\sigma^{\prime}}\right)_{\omega^{-1}}\left(h_{L_{0}}^{\sigma_{0}}, h_{\omega, Q}^{\sigma}\right)_{\omega}
$$


and

$$
\left(h_{L}^{\sigma_{0}}, h_{\omega^{-1}, Q^{\prime}}^{\sigma^{\prime}}\right)_{\omega^{-1}}=0,
$$

unless $L \subseteq Q^{\prime}$ and $\left(h_{L_{0}}^{\sigma_{0}}, h_{\omega, Q}^{\sigma}\right)_{\omega}=0$, unless $L_{0} \subseteq Q$. Hence we only have nonzero terms if $Q \subseteq Q^{\prime}$ or $\hat{Q}^{\prime} \subseteq Q$. Here $\hat{Q}^{\prime}$ is the parent of $Q^{\prime}$. This reflects the nice 'local' property of the shift operator. Even in the weighted setting we do not have terms for intervals that are far apart. Hence we can split the sum (4.3) into three parts, $\sum_{\hat{Q}^{\prime} \subsetneq Q}, \sum_{Q \subsetneq Q^{\prime}}$, and the diagonal part $\sum_{Q=Q^{\prime}}+\sum_{Q=\hat{Q}^{\prime}}$. We also sum over all corresponding signatures. We have turned our estimate into a dyadic tree estimate. From here on, we will be using certain imbedding theorems to reduce the estimate further to an inequality which reflects the norm of the shifts on certain test functions. To be precise, let us introduce the truncated shift $\amalg^{Q}: f \mapsto \sum_{L \subset Q}\left(f, h_{L}^{\sigma_{0}}\right) h_{L_{0}}^{\sigma_{0}}$ and its composition with multiplication by $\omega^{-1}$, $\amalg_{\omega^{-1}}^{Q}$. It will be crucial to know the weighted norm $\left\|\amalg_{\omega^{-1}}^{Q} 1\right\|_{\omega}$. Here $\amalg_{\omega^{-1}}^{Q} 1$ is well-defined in the sense that $\amalg_{\omega^{-1}}^{Q} 1=\amalg_{\omega^{-1}}^{Q} \chi_{K}$ for all $K \supseteq Q$. We are going to reduce our estimates with ease to the lemma below, which will be proven in Section 5 .

Lemma 4.2. We have the estimate $\left\|\amalg_{\omega^{-1}}^{Q} 1\right\|_{\omega} \lesssim Q_{2}(\omega) \omega^{-1}(Q)^{1 / 2}$ with the implied constant independent of the dyadic cube $Q$ and weight $\omega \in A_{2}$.

4.1. The reduction to shifts on characteristic functions. As for the diagonal part $\sum_{Q=\hat{Q}^{\prime}}+\sum_{Q=Q^{\prime}}$ of (4.3), it suffices to prove the two estimates

$$
\left|\left(\amalg_{\omega^{-1}} h_{\omega^{-1}, \hat{Q}^{\prime}}^{\sigma_{1}}, h_{\omega, Q^{\prime}}^{\sigma_{2}}\right)_{\omega}\right| \leq c Q_{2}(\omega)
$$

and

$$
\left|\left(\amalg_{\omega^{-1}} h_{\omega^{-1}, Q^{\prime}}^{\sigma_{1}}, h_{\omega, Q^{\prime}}^{\sigma_{2}}\right)_{\omega}\right| \leq c Q_{2}(\omega)
$$

for any choices of signatures $\sigma_{1}, \sigma_{2}$. These are a straightforward reduction to Lemma 4.2 .

As for the two parts $Q \subsetneq Q^{\prime}$ or $\hat{Q}^{\prime} \subsetneq Q$, let us observe that $\amalg\left(\omega^{-1} h_{\omega^{-1}, Q}^{\sigma}\right)$ is supported by $Q$ and that $\amalg^{*}\left(\omega h_{\omega, Q^{\prime}}^{\sigma}\right)$ is supported by $\hat{Q}^{\prime}$. This is the essential strong local property of the shift operator. We consider the sum $\hat{Q}^{\prime} \subsetneq Q$ here; the other sum is similar. This reduction is almost identical to [12], but we include it due to its importance and for convenience.

$$
\begin{aligned}
& \left|\sum_{Q, Q^{\prime}: \hat{Q}^{\prime} \subsetneq Q} \sum_{\sigma, \sigma^{\prime}}\left(f, h_{\omega^{-1}, Q}^{\sigma}\right)_{\omega^{-1}}\left(g, h_{\omega, Q^{\prime}}^{\sigma^{\prime}}\right)_{\omega}\left(\amalg_{\omega^{-1}} h_{\omega^{-1}, Q}^{\sigma}, h_{\omega, Q^{\prime}}^{\sigma^{\prime}}\right)_{\omega}\right| \\
& =\left|\sum_{Q, Q^{\prime}: \hat{Q}^{\prime} \subsetneq Q} \sum_{\sigma, \sigma^{\prime}}\left(f, h_{\omega^{-1}, Q}^{\sigma}\right)_{\omega^{-1}}\left(g, h_{\omega, Q^{\prime}}^{\sigma^{\prime}}\right)_{\omega}\left(h_{\omega^{-1}, Q}^{\sigma}, \amalg^{*}\left(\omega h_{\omega, Q^{\prime}}^{\sigma^{\prime}}\right)\right)_{\omega^{-1}}\right| \\
& \stackrel{(1)}{=}\left|\sum_{Q^{\prime}} \sum_{\sigma^{\prime}} \sum_{Q: Q \supsetneq \hat{Q}^{\prime}} \sum_{\sigma}\left(f, h_{\omega^{-1}, Q}^{\sigma}\right)_{\omega^{-1}} h_{\omega^{-1}, Q}^{\sigma}\left(\hat{Q}^{\prime}\right)\left(g, h_{\omega, Q^{\prime}}^{\sigma^{\prime}}\right)_{\omega}\left(\amalg_{\omega^{-1}} \chi_{\hat{Q}^{\prime}}, h_{\omega, Q^{\prime}}^{\sigma^{\prime}}\right)_{\omega}\right| \\
& \quad \stackrel{(2)}{\leq} \sum_{Q^{\prime}} \sum_{\sigma^{\prime}}\langle f\rangle_{\hat{Q}^{\prime}, \omega^{-1}}\left|\left(g, h_{\omega, Q^{\prime}}^{\sigma^{\prime}}\right)_{\omega}\left(\amalg_{\omega^{-1}} \chi_{\hat{Q}^{\prime}}, h_{\omega, Q^{\prime}}^{\sigma^{\prime}}\right)_{\omega}\right| \\
& \leq\|g\|_{\omega}\left(\sum_{Q^{\prime}} \sum_{\sigma^{\prime}}\langle f\rangle_{\hat{Q}^{\prime}, \omega^{-1}}^{2}\left(\amalg_{\omega^{-1}} \chi_{\hat{Q}^{\prime}}, h_{\omega, Q^{\prime}}^{\sigma^{\prime}}\right)_{\omega}^{2}\right)^{1 / 2},
\end{aligned}
$$


where (1) uses the fact that $\amalg^{*}\left(\omega h_{\omega, Q^{\prime}}\right)$ is supported by $\hat{Q}^{\prime}$ (recall that the notation $h_{\omega^{-1}, Q}^{\sigma}\left(\hat{Q}^{\prime}\right)$ means the value of $h_{\omega^{-1}, Q}^{\sigma}$ on $\left.\hat{Q}^{\prime}\right)$ and (2) uses (3.2). To prove the inequality

$$
\sum_{Q^{\prime}} \sum_{\sigma^{\prime}}\langle f\rangle_{\hat{Q}^{\prime}, \omega^{-1}}^{2}\left(\amalg_{\omega^{-1}} \chi_{\hat{Q}^{\prime}}, h_{\omega, Q^{\prime}}^{\sigma^{\prime}}\right)_{\omega}^{2} \leq c Q_{2}(\omega)^{2}\|f\|_{\omega^{-1}}^{2},
$$

we will apply a multi-variable version of a weighted Carleson imbedding theorem from $[10]$.

Theorem 4.3. Let $\alpha_{Q^{\prime}}^{\sigma^{\prime}}$ be a non-negative sequence such that for all dyadic $Q$

$$
\sum_{Q^{\prime} \subseteq Q} \sum_{\sigma^{\prime}} \alpha_{Q^{\prime}}^{\sigma^{\prime}} \leq A \omega^{-1}(Q)
$$

Then for all $f \in L^{2}\left(\omega^{-1}\right)$

$$
\sum_{Q^{\prime}} \sum_{\sigma^{\prime}} \alpha_{Q^{\prime}}^{\sigma^{\prime}}\langle f\rangle_{Q^{\prime}, \omega^{-1}}^{2} \lesssim A\|f\|_{\omega^{-1}}^{2}
$$

This theorem was proven in $\mathbb{R}$ using Bellman functions. One can use the same Bellman function technique, slightly modifying the dynamics condition by applying it to several variables to obtain the result. The new dynamics condition can be obtained by iteration and its derivation should present little difficulty for the reader familiar with this material. Our modified Haar system here makes this passage particularly convenient. To use the imbedding Theorem 4.3 in order to prove inequality (4.4), we shift the indices, so condition (4.5) in our situation becomes

$$
\sum_{Q^{\prime} \subsetneq Q} \sum_{\sigma^{\prime}}\left(\amalg_{\omega^{-1}} \chi_{\hat{Q}^{\prime}}, h_{\omega, Q^{\prime}}^{\sigma^{\prime}}\right)_{\omega}^{2} \leq c Q_{2}(\omega)^{2} \omega^{-1}(Q) .
$$

Here $\left(\amalg_{\omega^{-1}} \chi_{\hat{Q}^{\prime}}, h_{\omega, Q^{\prime}}^{\sigma^{\prime}}\right)_{\omega}=\sum_{L}\left(\omega^{-1} \chi_{\hat{Q}^{\prime}}, h_{L}^{\sigma_{0}}\right)\left(h_{L_{0}^{\sigma_{0}}}, h_{\omega, Q^{\prime}}^{\sigma^{\prime}}\right)_{\omega}$ and since $Q^{\prime} \subsetneq Q$, it is sufficient to consider only $L \subseteq Q$ because all other terms are zero. So the sum above is controlled by $\left\|\amalg_{\omega^{-1}}^{Q} 1\right\|_{\omega}^{2}$ and we reduced the sum $\sum_{{\hat{Q^{\prime}}}^{\prime} Q}$ to Lemma 4.2.

\section{THE NORM OF THE SHIFT ON CHARACTERISTIC FUNCTIONS}

We turn to the proof of Lemma 4.2, the essential part of this paper.

Proof. We estimate this norm by duality. It is sufficient to prove the inequality

$$
\left|\left(\amalg_{\omega^{-1}}^{Q} 1, f\right)_{\omega}\right| \leq c Q_{2}(\omega) \omega^{-1}(Q)^{1 / 2}\|f\|_{\omega}
$$


for positive test functions $f$ and cubes $Q$.

$$
\begin{aligned}
\left|\left(\amalg_{\omega^{-1}}^{Q} 1, f\right)_{\omega}\right|= & \left|\sum_{L \subseteq Q}\left(\omega^{-1}, h_{L}^{\sigma_{0}}\right) \sum_{Q^{\prime}} \sum_{\sigma^{\prime}}\left(f, h_{\omega, Q^{\prime}}^{\sigma^{\prime}}\right)_{\omega}\left(h_{L_{0}}^{\sigma_{0}}, h_{\omega, Q^{\prime}}^{\sigma^{\prime}}\right)_{\omega}\right| \\
\leq & \left|\sum_{L \subseteq Q}\left(\omega^{-1}, h_{L}^{\sigma_{0}}\right) \sum_{Q^{\prime}: Q^{\prime} \supsetneq L} \sum_{\sigma^{\prime}}\left(f, h_{\omega, Q^{\prime}}^{\sigma^{\prime}}\right)_{\omega}\left(h_{L_{0}}^{\sigma_{0}}, h_{\omega, Q^{\prime}}^{\sigma^{\prime}}\right)_{\omega}\right| \\
& +\sum_{L \subseteq Q} \sum_{\sigma^{\prime}}\left(\left|\left(\omega^{-1}, h_{L}^{\sigma_{0}}\right)\left(f, h_{\omega, L_{0}}^{\sigma^{\prime}}\right)_{\omega}\left(h_{L_{0}}^{\sigma_{0}}, h_{\omega, L_{0}}^{\sigma^{\prime}}\right)_{\omega}\right|\right. \\
& \left.+\left|\left(\omega^{-1}, h_{L}^{\sigma_{0}}\right)\left(f, h_{\omega, L}^{\sigma^{\prime}}\right)_{\omega}\left(h_{L_{0}}^{\sigma_{0}}, h_{\omega, L}^{\sigma^{\prime}}\right)_{\omega}\right|\right) \\
\leq & \sum_{L}\left|\left(\omega^{-1}, h_{L}^{\sigma_{0}}\right)\right|\left|\left(\omega, h_{L_{0}}^{\sigma_{0}}\right)\right|\langle f\rangle_{L, \omega}\left\langle\chi_{Q}\right\rangle_{L, \omega^{-1}} \\
& +2^{n}\|f\|_{\omega}\left(\sum_{L \subseteq Q}\left(\omega^{-1}, h_{L}^{\sigma_{0}}\right)^{2}\langle\omega\rangle_{L}\right)^{1 / 2}
\end{aligned}
$$

The first inequality holds because summands are zero unless $L_{0} \subseteq Q^{\prime}$. For the other steps we used (3.2), a simple estimate for the weighted inner products and the fact that $\langle\omega\rangle_{L_{0}} \leq 2^{n}\langle\omega\rangle_{L}$. To proceed, we will also be using the sharp bound for a weighted dyadic square function. Recall that the dyadic square function is defined by $f \mapsto S f$ where

$$
S f(x):=\left(\sum_{I \in \mathcal{D}} \sum_{\sigma}\left|\left(f, h_{I}\right)\right|^{2} \frac{\chi_{I}(x)}{|I|}\right)^{1 / 2} .
$$

As an operator in $L^{2}(\omega)$, the norm of the dyadic square function is bounded linearly. See [6] for the following result in one dimension:

Theorem 5.1. There exists a constant $c$ so that for all $\omega \in A_{2}$ the square function $S: L_{\mathbb{R}}^{2}(\omega) \rightarrow L_{\mathbb{R}}^{2}(\omega)$ has operator norm $\|S\| \leq c Q_{2}(\omega)$.

The proof in [6] is via Bellman functions. It uses the weighted imbedding theorem plus some other dyadic tree estimate that involves differences of averages of the weights. In our situation, we need Theorem 4.3 and an $n$-variable version of the tree estimate. An iteration of the dynamics condition to adjust it to several variables is needed here too.

The estimate on the square function implies that

$$
\left\|S\left(\omega^{-1} \chi_{Q}\right)\right\|_{\omega}^{2} \lesssim Q_{2}(\omega)^{2} \omega^{-1}(Q) .
$$

Writing the weighted $L^{2}$ norm and discarding some non-negative terms on the left yield the inequality

$$
\sum_{L \subseteq Q} \sum_{\sigma}\left(\omega^{-1}, h_{L}^{\sigma_{0}}\right)^{2}\langle\omega\rangle_{L} \lesssim Q_{2}(\omega)^{2} \omega^{-1}(Q)
$$

This finishes the estimate of the second summand in (5.7). To estimate the first summand, we are going to apply a bilinear weighted imbedding theorem from [12]. 
Theorem 5.2. Let $\omega$ and $v$ be weights so that $\langle\omega\rangle_{Q}\langle v\rangle_{Q} \leq A$ for all cubes $Q$ and let $\alpha_{Q}^{\sigma}$ be a non-negative sequence so that the three estimates below hold for all $Q$ :

$$
\sum_{L \subseteq Q} \sum_{\sigma} \frac{\alpha_{L}^{\sigma}}{\langle\omega\rangle_{L}} \leq A v(Q), \quad \sum_{L \subseteq Q} \sum_{\sigma} \frac{\alpha_{L}^{\sigma}}{\langle v\rangle_{L}} \leq A \omega(Q), \quad \sum_{L \subseteq Q} \sum_{\sigma} \alpha_{L}^{\sigma} \leq A|Q| .
$$

Then

$$
\sum_{Q} \sum_{\sigma} \alpha_{Q}^{\sigma}\langle f\rangle_{Q, \omega}\langle g\rangle_{Q, v} \lesssim A\|f\|_{\omega}\|g\|_{v}
$$

for all $f \in L^{2}(\omega)$ and $g \in L^{2}(v)$.

This is the $n$-dimensional analog of the version that appeared in [12]. The changes in the proof to obtain the version needed here are similar to those done for Theorem 4.3.

We will now check the imbedding conditions appearing in Theorem 5.2 with $v=\omega^{-1}, \alpha_{L}^{\sigma_{0}}=\left|\left(\omega^{-1}, h_{L}^{\sigma_{0}}\right)\right|\left|\left(\omega, h_{L_{0}}^{\sigma_{0}}\right)\right|$ and $\alpha_{L}^{\sigma}=0$ if $\sigma \neq \sigma_{0}$. We have $A \sim Q_{2}(\omega)$.

5.1. Estimation of the imbedding conditions. We have to estimate three sums in the following manner:

$$
\begin{gathered}
\sum_{L \subseteq Q}\left|\left(\omega^{-1}, h_{L}^{\sigma_{0}}\right)\right|\left|\left(\omega, h_{L_{0}}^{\sigma_{0}}\right)\right| \frac{1}{\langle\omega\rangle_{L}} \lesssim Q_{2}(\omega) \omega^{-1}(Q), \\
\sum_{L \subseteq Q}\left|\left(\omega^{-1}, h_{L}^{\sigma_{0}}\right)\right|\left|\left(\omega, h_{L_{0}}^{\sigma_{0}}\right)\right| \frac{1}{\left\langle\omega^{-1}\right\rangle_{L}} \lesssim Q_{2}(\omega) \omega(Q), \\
\sum_{L \subseteq Q}\left|\left(\omega^{-1}, h_{L}^{\sigma_{0}}\right)\right|\left|\left(\omega, h_{L_{0}}^{\sigma_{0}}\right)\right| \lesssim Q_{2}(\omega)|Q| .
\end{gathered}
$$

For conditions (5.9) and (5.10) we use the Cauchy-Schwartz inequality to separate the differences in $\omega$ and $\omega^{-1}$. There is not much difference to the one-dimensional case. We just use a simple $n$-dimensional analog of the dynamics in the corresponding Bellman proofs to estimate the sums exactly as done in [12].

Estimating condition (5.11) is the main difficulty here. The sum is bilinear and does not split into a product of two sums separating the differences in $\omega$ and $\omega^{-1}$ as in the previous two sums. The difficulty is to estimate the product of differences of averages of $\omega$ and $\omega^{-1}$ over intervals that are a pair of parent $L$ and child $L_{0}$. So it is necessary that the Bellman function carry additionally the information of the averages of $\omega$ over all of the $2^{n}$ children of a cube as its variables. A delicate dynamics condition is required. To reduce the strain on notation due to the large number of variables involved for general $n$, we demonstrate how this can be done for $n=2$ first and then pass on to describe the procedure for general $n$. Without loss of generality, we fix the shift operator here so that $L_{0}$ is the northwest corner child of $L$.

Proof. As in [12] we consider for any constant $Q$ the function

$$
B(u, v)=Q^{3 / 4} \sqrt[4]{u v}
$$


on the first quadrant $\mathcal{T}=\left(\mathbb{R}_{+}\right)^{2}$ and its restriction to the domain $\mathcal{S}=\{(u, v) \in$ $\left.\mathbb{R}_{+}^{2}: 0 \leq u v \leq 4 Q\right\}$. Then $B$ has the properties

$$
\begin{gathered}
(u, v) \in \mathcal{S} \Longrightarrow 0 \leq B(u, v) \leq 4 Q \\
(u, v) \in \mathcal{T} \Longrightarrow \frac{Q^{3 / 4}}{8} D(u, v) \leq-d^{2} B(u, v) \leq \frac{Q^{3 / 4}}{4} D(u, v),
\end{gathered}
$$

where $d^{2} B(u, v)$ denotes the Hessian of $B$ at $(u, v)$ and $D(u, v)$ is the diagonal operator defined by

$$
D(u, v)=u^{-7 / 4} v^{1 / 4}(d u)^{2}+u^{1 / 4} v^{-7 / 4}(d v)^{2} .
$$

Inequality (5.13) is to be understood in terms of operators. The size estimate (5.12) is easy to check using the condition $u v \leq 4 Q$. The diagonal property of the second derivative, property (5.13) can be seen by directly computing the second derivative.

Applying Taylor's theorem together with (5.13) allows us to conclude that there are functions $a_{i}, b_{i}$ so that

$$
\begin{aligned}
& B(u, v)+\nabla B(u, v)(\Delta u, \Delta v)^{t}-a_{1}(u, v)(\Delta u)^{2}-a_{2}(u, v)(\Delta v)^{2} \\
\leq & B(u+\Delta u, v+\Delta v) \\
\leq & B(u, v)+\nabla B(u, v)(\Delta u, \Delta v)^{t}-b_{1}(u, v)(\Delta u)^{2}-b_{2}(u, v)(\Delta v)^{2} .
\end{aligned}
$$

If $1 \leq u v \leq Q$ and $-u \leq \Delta u \leq 15 u$ and $-v \leq \Delta v \leq 15 v$, then we can use the functions

$$
\begin{array}{rlrl}
a_{1}(u, v)=16^{1 / 4} Q^{3 / 4} u^{-7 / 4} v^{1 / 4}, & & a_{2}(u, v)=16^{1 / 4} Q^{3 / 4} u^{1 / 4} v^{-7 / 4}, \\
b_{1}(u, v)=16^{-7 / 4} 18^{-1} Q^{3 / 4} u^{-7 / 4} v^{1 / 4}, & b_{2}(u, v)=16^{-7 / 4} 18^{-1} Q^{3 / 4} u^{1 / 4} v^{-7 / 4} .
\end{array}
$$

So the ratios are bounded above by $a_{i}(u, v) / b_{i}(u, v) \leq 256$ and products are bounded below by $b_{1}(u, v) b_{2}(u, v) \geq 16^{-7 / 2} \cdot 18^{-2}$. Let us define

$$
\begin{aligned}
& \mathbf{b}\left(u, v, \Delta_{1} v, \Delta_{2} v_{+}, \Delta_{2} v_{-}\right)=10000 B(u, v) \\
& \quad+\quad B\left(u, v+\Delta_{1} v+\Delta_{2} v_{+}\right)+B\left(u, v+\Delta_{1} v-\Delta_{2} v_{+}\right) \\
& \quad+\quad B\left(u, v-\Delta_{1} v+\Delta_{2} v_{-}\right)+B\left(u, v-\Delta_{1} v-\Delta_{2} v_{-}\right)
\end{aligned}
$$

on the following subset $\mathcal{O}$ of $\mathbb{R}^{5}:\left(u, v, \Delta_{1} v, \Delta_{2} v_{+}, \Delta_{2} v_{-}\right) \in \mathcal{O}$ if all five pairs $(u, v),\left(u, v+\Delta_{1} v+\Delta_{2} v_{+}\right),\left(u, v+\Delta_{1} v-\Delta_{2} v_{+}\right),\left(u, v-\Delta_{1} v+\Delta_{2} v_{-}\right),\left(u, v-\Delta_{1} v-\right.$ $\left.\Delta_{2} v_{-}\right) \in \mathcal{S}$. We write for example $v^{n w}$ for $v+\Delta_{1} v+\Delta_{2} v_{+}$because it will correspond to the average of the northwest corner square. Using this notation, we have

$$
\begin{aligned}
& \mathbf{b}\left(u, v, \Delta_{1} v, \Delta_{2} v_{+}, \Delta_{2} v_{-}\right) \\
& \quad=10000 B(u, v)+B\left(u, v^{n w}\right)+B\left(u, v^{s w}\right)+B\left(u, v^{n e}\right)+B\left(u, v^{s e}\right) .
\end{aligned}
$$

Here $\mathbf{b}$ has the size estimate

$$
\left(u, v, \Delta_{1} v, \Delta_{2} v_{+}, \Delta_{2} v_{-}\right) \in \mathcal{O} \Longrightarrow 0 \leq \mathbf{b}\left(u, v, \Delta_{1} v, \Delta_{2} v_{+}, \Delta_{2} v_{-}\right) \leq 50000 Q
$$

and the dynamics inequality

$$
\begin{aligned}
4 \mathbf{b}\left(u, v, \Delta_{1} v, \Delta_{2} v_{+}, \Delta_{2} v_{-}\right)-c\left|\Delta_{1} u\right|\left|\Delta_{1} v^{n w}\right| \\
\geq
\end{aligned}
$$


with some fixed positive $c$, provided that all five vectors that are plugged into $\mathbf{b}$ here belong to $\mathcal{O}$.

The condition on the size (5.16) is inherited from (5.12), the size estimate for $B$. Let us show the main inequality (5.17) which will give us the correct dynamics for a Bellman function argument. We will use inequality (5.15) with $\Delta u=0$ and $\Delta v$ so that $v+\Delta v=v^{*}$. Here $*$ stands interchangeably for $n w, s w$, se or ne. Note that $-v \leq \Delta v \leq 3 v$. The gradient terms will cancel in each case and the mixed terms arising from the squared terms also cancel. We first estimate the left hand side of (5.17) from below:

$$
\begin{aligned}
& 4 \mathbf{b}\left(u, v, \Delta_{1} v, \Delta_{2} v_{+}, \Delta_{2} v_{-}\right)-c\left|\Delta_{1} u\right|\left|\Delta_{1} v^{n w}\right| \\
& \quad \geq 40016 B(u, v)-16 a_{2}\left(\Delta_{1} v\right)^{2}-8 a_{2}\left(\Delta_{2} v_{+}\right)^{2}-8 a_{2}\left(\Delta_{2} v_{-}\right)^{2}-c\left|\Delta_{1} u \| \Delta_{1} v^{n w}\right| .
\end{aligned}
$$

We turn to an upper estimate for the right hand side of (5.17). In the following, we again use inequality (5.15) several times, applying it to $(u, v)$ with $\Delta u$ 's and $\Delta v$ 's so that $u+\Delta u=u^{*}$ and $v+\Delta v=v^{*^{*}}$. Still, $-v \leq \Delta v \leq 15 v$ and similarly for $u$. We will drop some negative squared terms here and observe that the gradient terms and mixed squared terms cancel.

$$
\begin{aligned}
& \mathbf{b}\left(u^{n w}, v^{n w}, \Delta_{1} v^{n w}, \Delta_{2} v_{+}^{n w}, \Delta_{2} v_{-}^{n w}\right)+\mathbf{b}\left(u^{n e}, v^{n e}, \Delta_{1} v^{n e}, \Delta_{2} v_{+}^{n e}, \Delta_{2} v_{-}^{n e}\right) \\
& \quad+\mathbf{b}\left(u^{s w}, v^{s w}, \Delta_{1} v^{s w}, \Delta_{2} v_{+}^{s w}, \Delta_{2} v_{-}^{s w}\right)+\mathbf{b}\left(u^{s e}, v^{s e}, \Delta_{1} v^{s e}, \Delta_{2} v_{+}^{s e}, \Delta_{2} v_{-}^{s e}\right) \\
& \leq \quad 40016 B(u, v)-40016 b_{1}\left(\Delta_{1} u\right)^{2}-40016 b_{2}\left(\Delta_{1} v\right)^{2} \\
& \quad-20008 b_{2}\left(\Delta_{2} v_{+}\right)^{2}-20008 b_{2}\left(\Delta_{2} v_{-}\right)^{2}-4 b_{2}\left(\Delta_{1} v^{n w}\right)^{2} \\
& \leq \quad 40016 B(u, v)-800 \sqrt{b_{1} b_{2}}\left|\Delta_{1} u \| \Delta_{1} v^{n w}\right| \\
&-40016 b_{2}\left(\Delta_{1} v\right)^{2}-20008 b_{2}\left(\Delta_{2} v_{+}\right)^{2}-20008 b_{2}\left(\Delta_{2} v_{-}\right)^{2} .
\end{aligned}
$$

Combining these two estimates proves inequality (5.17) because of the lower bound on $b_{1}(u, v) b_{2}(u, v)$ and the upper bound on the ratio $a_{2}(u, v) / b_{2}(u, v)$. We point out that it is essential that the functions $a_{i}$ and $b_{i}$ depend upon the same $u$ and $v$ so that $1 \leq u v \leq Q$ for otherwise we do not have the control on their ratios. While it appears to be possible to use the dynamics inequality from [12] together with some additional considerations on Bellman functions to derive this dynamics condition, we find the proof presented here more streamlined and self-contained.

We are now ready to run the Bellman function proof. We fix $Q$ and plug into $B$ averages of the weight $\omega$ and its inverse. For example, $\langle\omega\rangle_{Q}$ will be plugged in for $v,\left\langle\omega^{-1}\right\rangle_{Q}$ plays the role of $u$, and $\Delta_{1, Q} \omega:=1 / 2\left(\langle\omega\rangle_{Q_{e}}-\langle\omega\rangle_{Q_{w}}\right)$ is $\Delta_{1} v$, the difference of the average over the east and west half of the cube $Q$. Moreover, $\Delta_{2}$ means taking the difference of the average in the other direction, i.e. north-south over the east and west sub-rectangles of $Q$. Note that the $A_{2}$ condition means that such quintuplets are in our domain $\mathcal{O}$ for any choice of cube, if we pick $Q=Q_{2}(\omega)$. Then the main inequality (5.17) yields the tree dynamics needed: using the size estimate (5.16) of $\mathbf{b}$ and going $N$ steps down yield the inequality

$$
\begin{aligned}
50000 Q_{2}(\omega)|Q| \geq & \sum_{L \subseteq Q,|L|=4^{-N}|Q|}|L| \mathbf{b}\left(\left\langle\omega^{-1}\right\rangle_{L},\langle\omega\rangle_{L}, \Delta_{1, L} \omega, \Delta_{2, L_{w}} \omega, \Delta_{2, L_{e}} \omega\right) \\
& +c \sum_{L \subseteq Q,|L|>4^{-N}|Q|}|L|\left|\Delta_{1, L^{2}} \omega^{-1}\right|\left|\Delta_{1, L_{n w}} \omega\right| .
\end{aligned}
$$

Discarding non-negative terms and letting $N \rightarrow \infty$ yield the desired estimate (5.11) and complete the proof of the lemma for $n=2$. For general $n$, we have to adjust the 
domain to $u v \leq 2^{n} Q$ and modify the definition of $\mathbf{b}$. The function $\mathbf{b}$ will depend upon $u$ and $\sum_{k=0}^{n-1} 2^{k}=2^{n}-1$ versions of $v$ at different scales. The coefficient of 10000 in the definition will have to be increased and hence the upper estimates of $\mathbf{b}$ will change as well as the functions $a_{i}$ and $b_{i}$ used in inequality (5.15).

This finishes the estimate of the imbedding conditions.

Having checked all imbedding conditions for Theorem 5.2, we finished the proof of Lemma 4.2 .

\section{The $L^{p}$ CASE AND SHARPNESS OF The RESUlt}

To obtain the norm estimate in Corollary 2.2 from Theorem 2.1, one employs the linear bound for $p=2$ together with the extrapolation theorem below, which is a special case of the result contained in [4]:

Theorem 6.1. If $T$ is a linear operator, defined in the appropriate spaces, and $N_{r}$ is an increasing function so that $\|T\|_{L^{r}(\omega)} \lesssim N_{r}\left(Q_{r}(\omega)\right)$ for all weights $\omega \in A_{r}$, then we have the estimate $\|T\|_{L^{p}(\omega)} \lesssim N_{p}\left(Q_{p}(\omega)\right)$ for all $\omega \in A_{p}$, where

$$
N_{p}(x) \lesssim \begin{cases}N_{r}(x), & p \geq r \\ N_{r}(x)^{\frac{r-1}{p-1}}, & p<r .\end{cases}
$$

Using the estimate for $p=2$ from Theorem 2.1 and applying Theorem 6.1 to $r=2$ yield the linear bound for $p>2$ and a power of $\frac{1}{p-1}=\frac{p^{\prime}}{p}$ for $p<2$. This establishes the bound claimed in Corollary 2.2.

To demonstrate sharpness of our estimates, it is necessary to treat all $p$ separately. We show sharpness for $p \leq 2$ by a family of examples and deduce the range of other $p$ by duality. As mentioned before, we consider power weights. For $p \leq 2$ and any $s \in(0,1)$ pick the family of weights $\omega_{s}(x)=|x|^{(n-s)(p-1)}$. One can see that these weights are in the class $A_{p}$ and that $Q_{p}\left(\omega_{s}\right) \leq s^{1-p}$ and hence $Q_{p}\left(\omega_{s}\right)^{p^{\prime} / p} \leq s^{-1}$. We pick a family of functions $f=x^{s-n} \chi_{(0,1]^{n}}$ and see that $\left\|f_{s}\right\|_{\omega_{s}} \lesssim s^{-1 / p}$. For example, to show sharpness for $R_{1}$, we aim to estimate $\left\|R_{1} f_{s}\right\|_{\omega_{s}} \geq s^{-(1+1 / p)}$ and we let $s \rightarrow 0$ to see that $Q_{p}^{p^{\prime} / p}$ is the best possible. To do so, let us consider the set $A_{1}=\left\{y: y_{1}>2, y_{i}<y_{1}, i=2, \ldots, n\right\}$. For $y \in A_{1}$ and $x \in(0,1]^{n}$ we have $\frac{\left(y_{1}-x_{1}\right)}{|y-x|} \gtrsim 1$ and therefore $\frac{\left(y_{1}-x_{1}\right)}{|y-x|^{n+1}} \gtrsim \frac{1}{|y|^{n}}$. So we estimate

$$
\begin{aligned}
\left\|R_{1} f_{s}\right\|_{\omega_{s}}^{p} & \geq\left.\left.\int_{A_{1}}\left|\int_{(0,1]^{n}} \frac{y_{1}-x_{1}}{|y-x|^{n}}\right| x\right|^{s-n} d A(x)\right|^{p}|y|^{(n-s)(p-1)} d A(y) \\
& \gtrsim \int_{A_{1}}\left|\int_{0}^{1} r^{s-n} r^{n-1} d r\right|^{p}|y|^{(n-s)(p-1)-p n} d A(y) \\
& \gtrsim s^{-p} \int_{2 \sqrt{n}}^{\infty} r^{-s p-n+s} r^{n-1} d r \gtrsim s^{-p-1},
\end{aligned}
$$

which establishes sharpness for $1<p<2$. For $p>2$, observe that $R_{i}^{*}=-R_{i}$ and that the adjoint operator $R_{i}^{*}$ is bounded on the dual space $L^{p}(\omega)^{*}=L^{p^{\prime}}\left(\omega^{1-p^{\prime}}\right)$ with the same bound, i.e. $\left\|R_{i}\right\|_{L^{p}(\omega)}=\left\|R_{i}^{*}\right\|_{L^{p^{\prime}}\left(\omega^{1-p^{\prime}}\right)}$. So in particular, we have $\left\|R_{i}\right\|_{L^{p}(\omega)}=\left\|R_{i}\right\|_{L^{p^{\prime}\left(\omega^{1-p^{\prime}}\right)}}$. Observe also that $Q_{p}\left(\omega^{1-p}\right)^{p^{\prime}-1}=Q_{p^{\prime}}(\omega)$. Assume a better than linear estimate for $p \geq 2$, say $N_{p}\left(Q_{p}(\omega)\right)$, where $N_{p}(x)$ grows slower than $x$ as $x \rightarrow \infty$. We already showed that for the dual exponent $p^{\prime}$ of $p$, since $1<p^{\prime}<2$, the optimal power is $p^{\prime \prime} / p^{\prime}=p / p^{\prime}$. Applying Theorem 6.1 for $r=p$ 
gives for the dual exponent $p^{\prime}<p$ the estimate $\left\|R_{i}\right\|_{L^{p^{\prime}}(\omega)}=\left\|R_{i}\right\|_{L^{p}\left(\omega^{1-p}\right)} \lesssim$ $N_{p}\left(Q_{p}\left(\omega^{1-p}\right)\right)=N_{p}\left(Q_{p^{\prime}}(\omega)^{\frac{1}{p^{\prime}-1}}\right)=N_{p}\left(Q_{p^{\prime}}(\omega)^{p / p^{\prime}}\right)$ which gives a growth better than we saw possible for this range of exponent. We have established sharpness of our result for all $p$.

\section{Remarks AND QUeSTIONS}

It is clear that our estimates do depend upon the dimension. The averaging of the Riesz transforms by means of Haar shifts already induces a dimensional constant. Also, we did not take care to avoid dimensional constants in the Bellman function proofs to estimate the Haar shifts themselves. This can be done, but it was, however, not relevant to our task as the choice of the underlying sets when defining the classical $A_{2}$ characteristic of course already changes the dimensional constants. It is, however, very interesting to ask for which version of $A_{2}$ the bound is independent of dimension. For squares of Riesz transforms, the correct version of $A_{2}$ is heat- $A_{2}$, where the box averages $\langle\omega\rangle_{Q}$ are replaced by heat averages $H \omega(x, t)$. One thus obtains a dimension-free weighted bound. The natural choice for single powers of Riesz transforms is Poisson averages. This is due to the availability of certain Littlewood Paley identities; see for example [15]. One obtains a linear and dimensionless bound, but for a class of weights which is for $n>1$ much smaller than classical $A_{2}$ : if $n>1$, there are power weights which are in the classical $A_{2}$ class for which the Poisson integral diverges. Still, the version of $A_{2}$ that makes use of the Poisson flow should be a good choice for weighted questions concerning first order Riesz transforms in very general settings.

\section{ACKNOWLEDGMENT}

The author is grateful to the Institute Henri Poincaré in Paris.

\section{REFERENCES}

[1] K. Astala, T. Imaniec, E. Saksman, Beltrami operators, Duke Math. J. 107 (2001), no. 1, pp. 27-56. MR1815249 (2001m:30021)

[2] S. Buckley, Summation condition on weights, Mich. Math. J. 40(1) (1993), pp. 153-170. MR1214060 (94d:42021)

[3] R. R. Coifman, C. Fefferman, Weighted norm inequalities for maximal functions and singular integrals, Studia Math. 51 (1974), pp. 241-250. MR0358205 (50:10670)

[4] O. Dragicević, L. Grafakos, M. C. Pereyra, S. Petermichl, Extrapolation and sharp norm estimates for classical operators on weighted Lebesgue spaces, Publ. Mat. 49 (2005), no. 1, pp. 73-91. MR2140200 (2006d:42019)

[5] R. Fefferman, C. Kenig, J. Pipher, The theory of weights and the Dirichlet problem for elliptic equations, Annals of Math. 134 (1991), pp. 65-124. MR1114608 (93h:31010)

[6] S. Hucovic, S. Treil, A. Volberg, The Bellman functions and the sharp square estimates for square functions, Operator Theory: Advances and Applications, the volume in memory of S. A. Vinogradov, v. 113, Birkhauser Verlag, 2000.

[7] R. A. Hunt, B. Muckenhoupt, R. L. Wheeden, Weighted norm inequalities for the conjugate function and the Hilbert transform, Trans. Amer. Math. Soc. 176 (1973), pp. 227-251. MR0312139 (47:701)

[8] A. LERNER, On some weighted norm inequalities for Littlewood-Paley operators, preprint 2006.

[9] B. Muckenhoupt, Weighted norm inequalities for the Hardy maximal function, Trans. Amer. Math. Soc. 165 (1972), pp. 207-226. MR0293384 (45:2461) 
[10] F. Nazarov, S. Treil, A. Volberg, The Bellman functions and two weight inequalities for Haar multipliers, J. of Amer. Math. Soc. 12 (1999), no. 4, pp. 909-928. MR1685781 (2000k:42009)

[11] S. Petermichl, Dyadic shifts and a logarithmic estimate for Hankel operators with matrix symbol, Comptes Rendus Acad. Sci. Paris, t. 330 (2000), no. 1, pp. 455-460. MR1756958 (2000m:42016)

[12] S. Petermichl, The sharp bound for the Hilbert transform in weighted Lebesgue spaces in terms of the classical $A_{p}$ characteristic, to appear in Amer. J. Math.

[13] S. Petermichl, S. Treil, A. Volberg, Why are the Riesz transforms averages of the dyadic shift?, Proceedings of the 6th international conference on harmonic analysis (El Escorial), Publ. Mat. (2002), Extra Vol., pp. 209-228. MR1964822 (2003m:42028)

[14] S. Petermichl, A. Volberg, Heating of the Beurling operator: weakly quasiregular maps on the plane are quasiregular, Duke Math. J. 112, No. 2 (2002), pp. 281-305. MR1894362 (2003d:42025)

[15] S. Petermichl, J. Wittwer, A sharp weighted estimate on the norm of Hilbert transform via invariant $A_{2}$ characteristic of the weight, Mich. Math. J. 50 (2002), pp. 71-87. MR1897034 (2003e:42016)

Institut de Mathématiques de Bordeaux, 351, Cours de la Libération, F-33405 Talence Cedex, France

E-mail address: Stefanie.Petermichl@math.u-bordeaux1.fr 